\title{
Node Filtering and Face Routing for Sensor Network
}

\section{Umang Amatya}

Department of Computer Science

\section{Main Contributions}

- Efficient Algorithms for identifying Redundant Sensor Nodes

New Technique for Filtering Redundant Nodes in Sensor Network

Reliable Algorithm for Message Routing Forwarding

- User Friendly Prototype Implementation in Java

- Results of Experimental Investigation

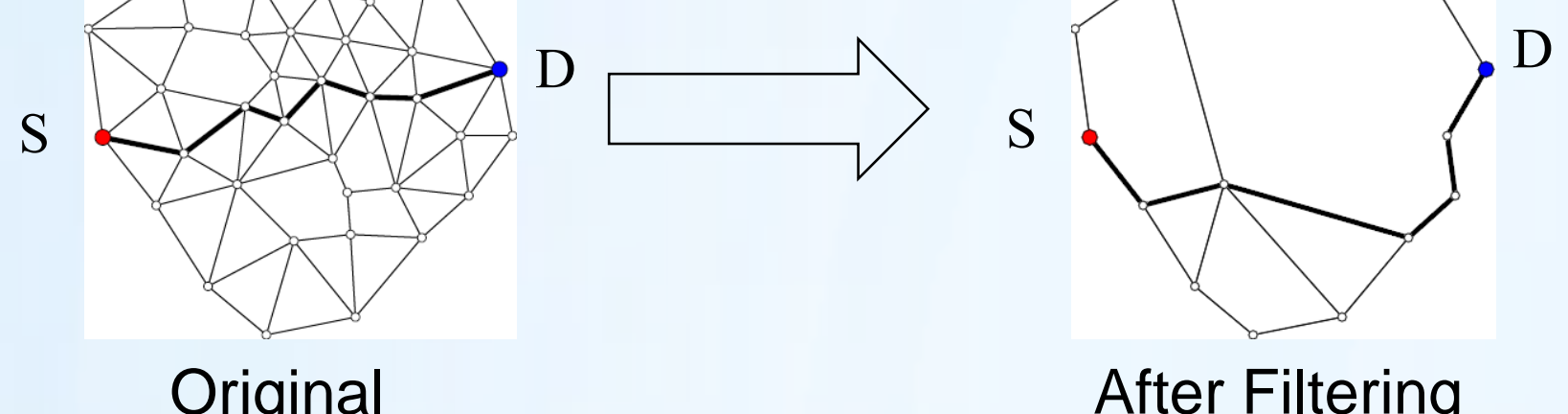

Original
What are Sensor Nodes?

Small scale electronic devices with

- Limited memory and processing power

- Wireless Communication Interface (range $\approx 70$ meters)

- Low-power Battery

- Sensing component (temperature, sound, ...)

Usually assumed to be stationary

Allowed to be active/inactive

Typical Sensor Nodes:

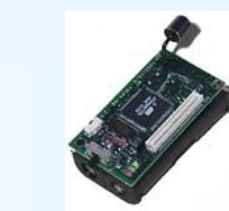

Berkeley MICA Mote

- Cost - $\$ 10$

- Weight $-70 \mathrm{~g}$

- Battery - 15k

Memory - 4KB Ram

OS - TinyOS

Radio Range - 30m

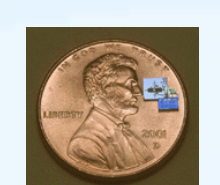

Smart Dus

- Cost < $\$ 1$

Size $-0.002 \mathrm{~cm}$

Weight -0.002

- Battery - Less

- Memory - Less

- OS - Smadio Range -

Radio Range 10
Shorter

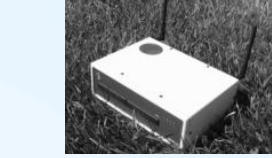

WINS NG 2.0 - Cost - $\$ 100$ - Size $-5300 \mathrm{~cm}^{3}$

- Weight $-5400 \mathrm{~g}$

- Battery - 300kj

- Memory - 32MB

- OS - Linux

Radio Range
Randomly Generated 500 Sensor-Nodes inside a Rectangular Region

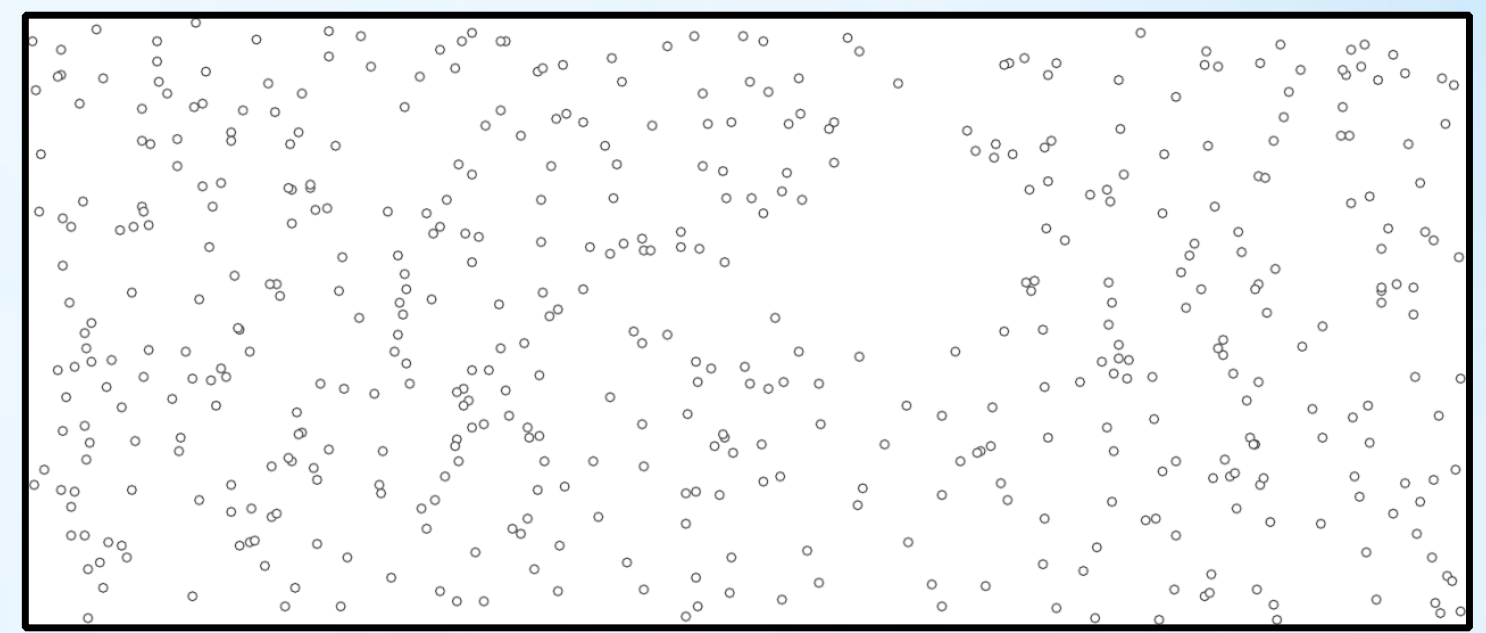

Randomly Generated 600 clustered Sensor-Nodes inside a Rectangular Region

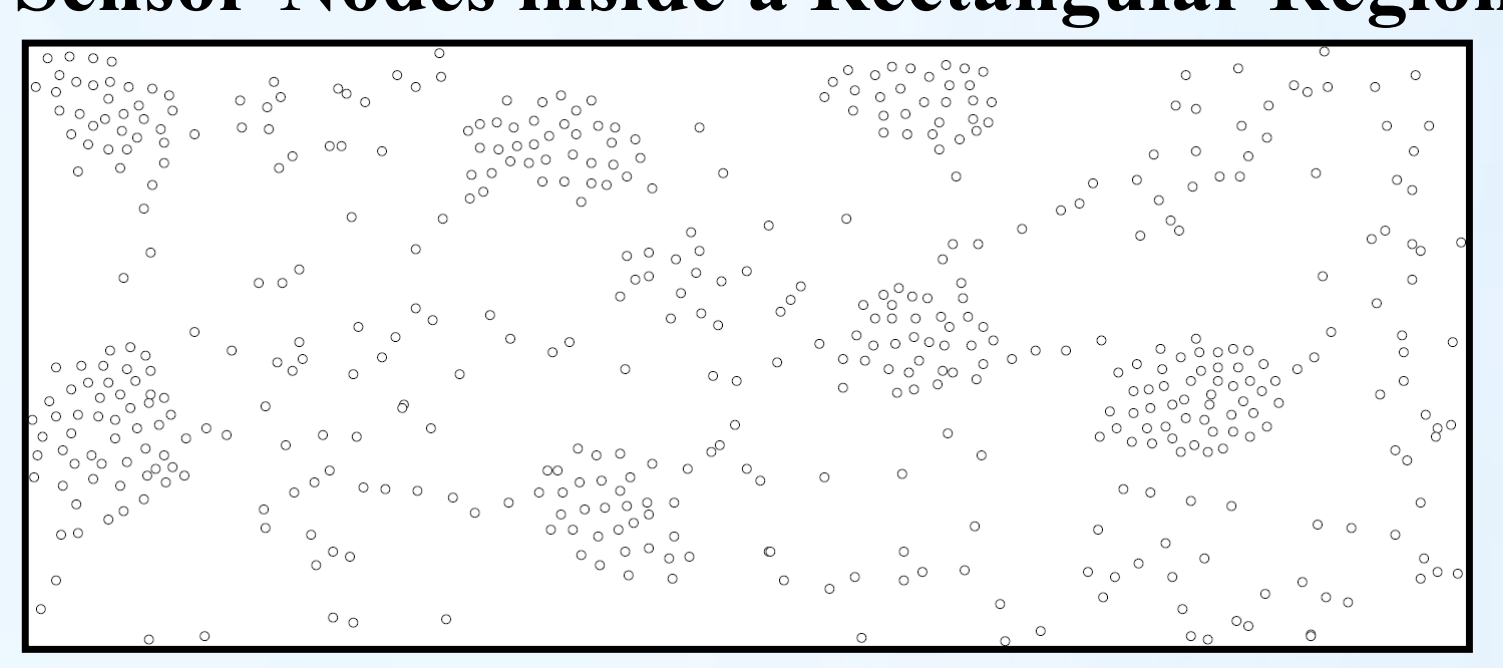

Sensor Network Modeled by Gabriel Net

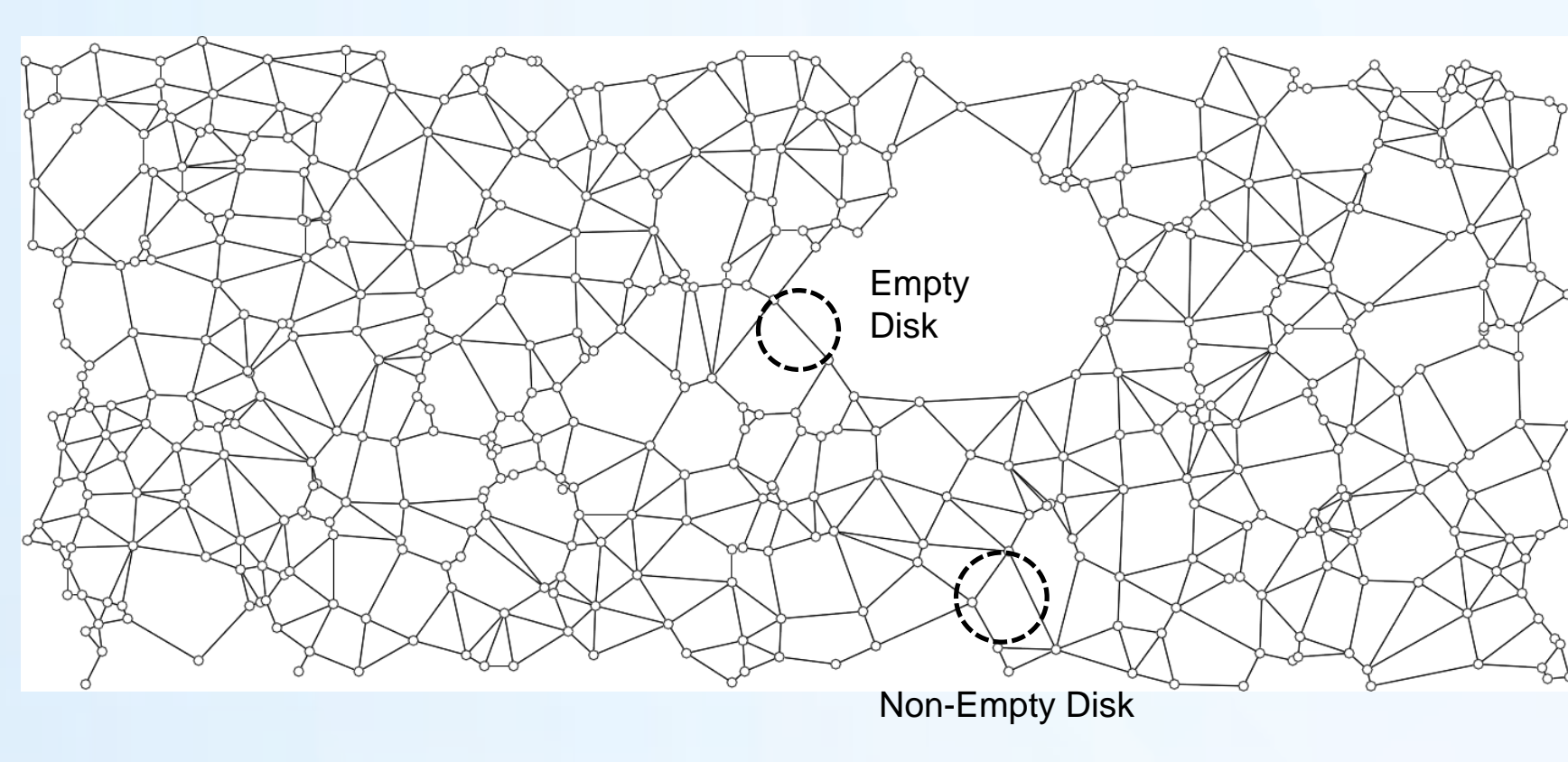

Linking Rule: Given a set of nodes $\mathrm{S}=\mathrm{p}_{0}, \mathrm{p}_{1}, \mathrm{p}_{2}, \ldots, \mathrm{p}_{\mathrm{n}-1}$, two nodes $p_{i}$ and $p_{j}$ are connected by an edge if the disk with diameter ending at $p_{i}$ and $p_{j}$ does not contain any node.

\section{Greedy Routing in Gabriel-Net}

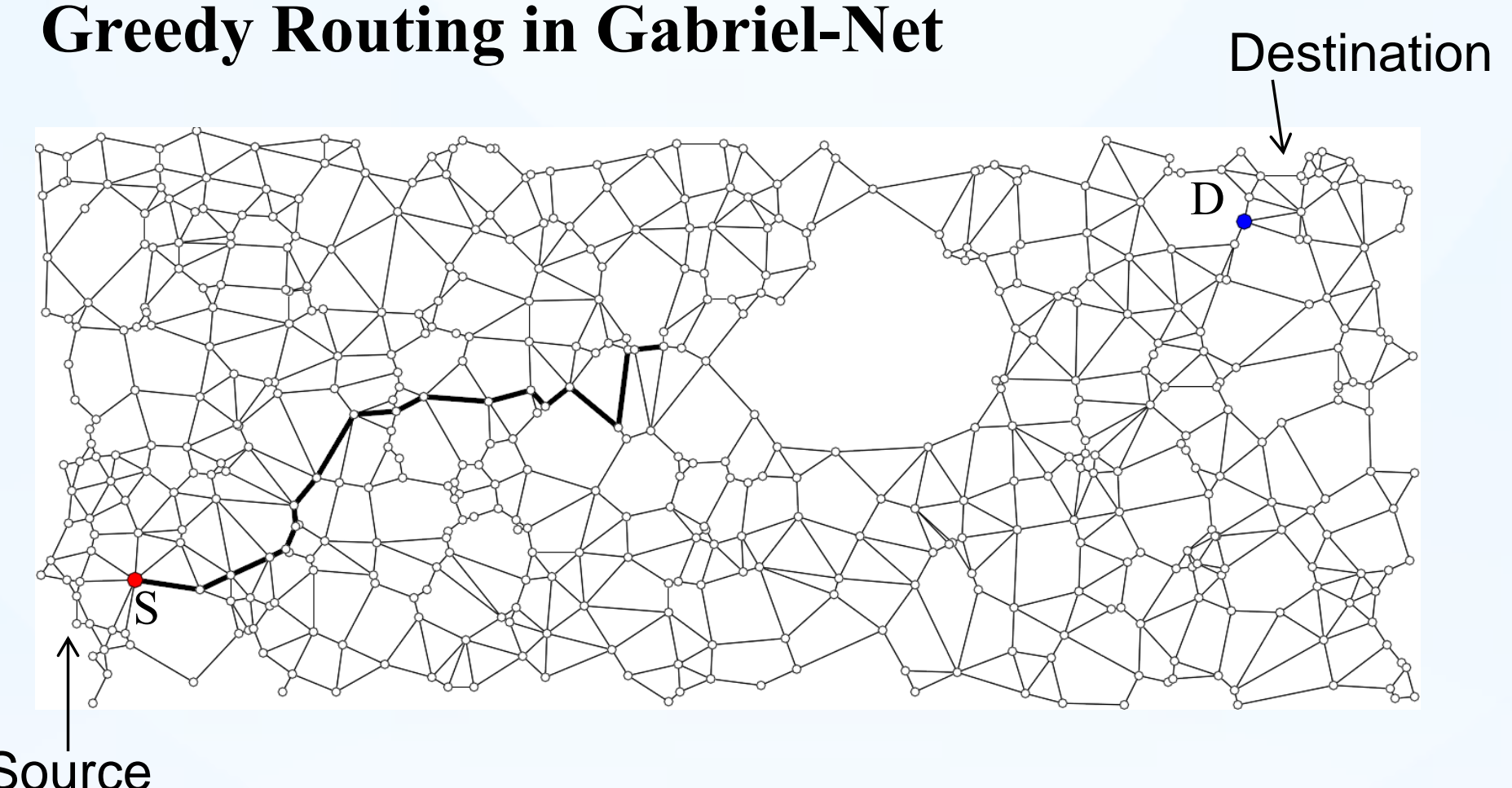

- How to send packet from the source(S) node to destination(D) node?

- Greedy Rule: Forward packet to the node closest to the destination node

- Thick-Segment Route (

\section{Occasional Trouble with Greedy Routing!}

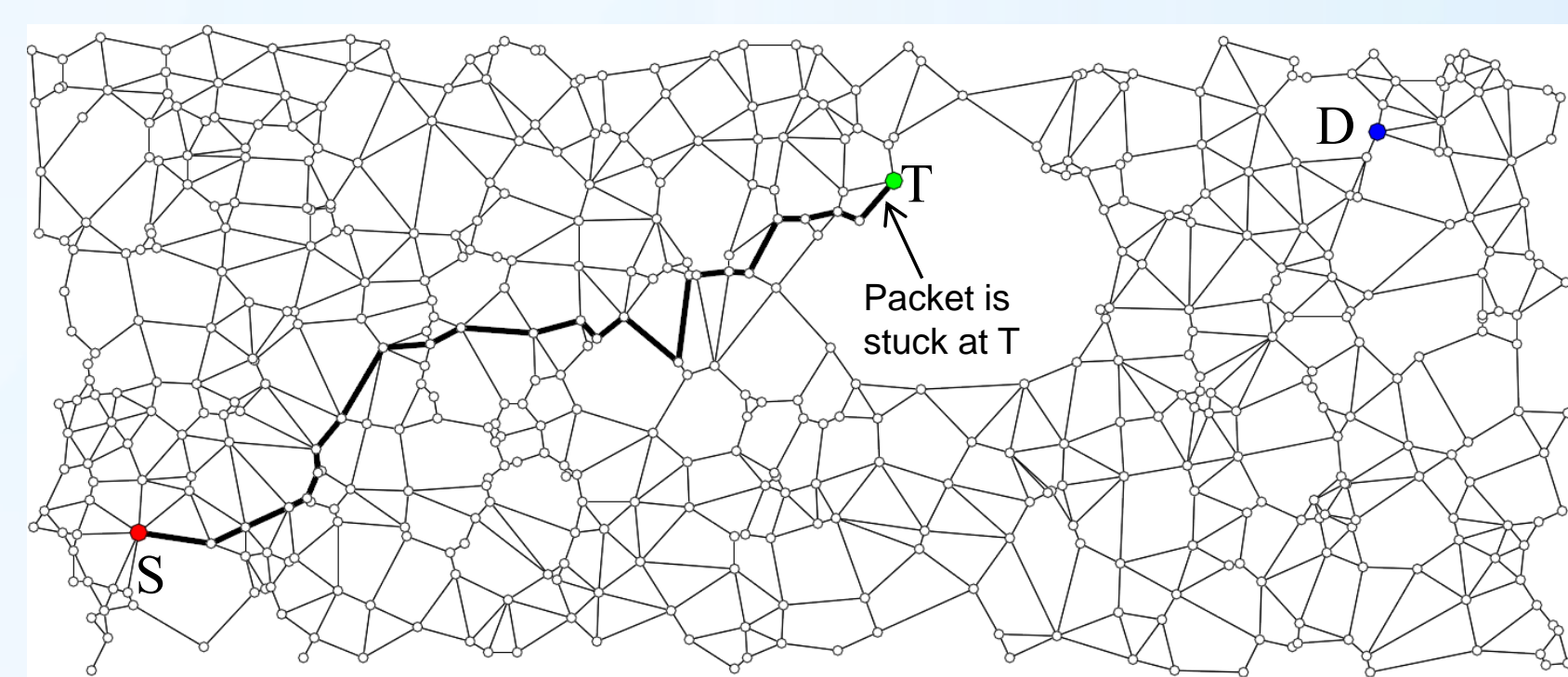

While sending packet from red node (S) to blue node (D), it get trapped in the green node $(\mathrm{T})$
How to Fix Trapping Problem?

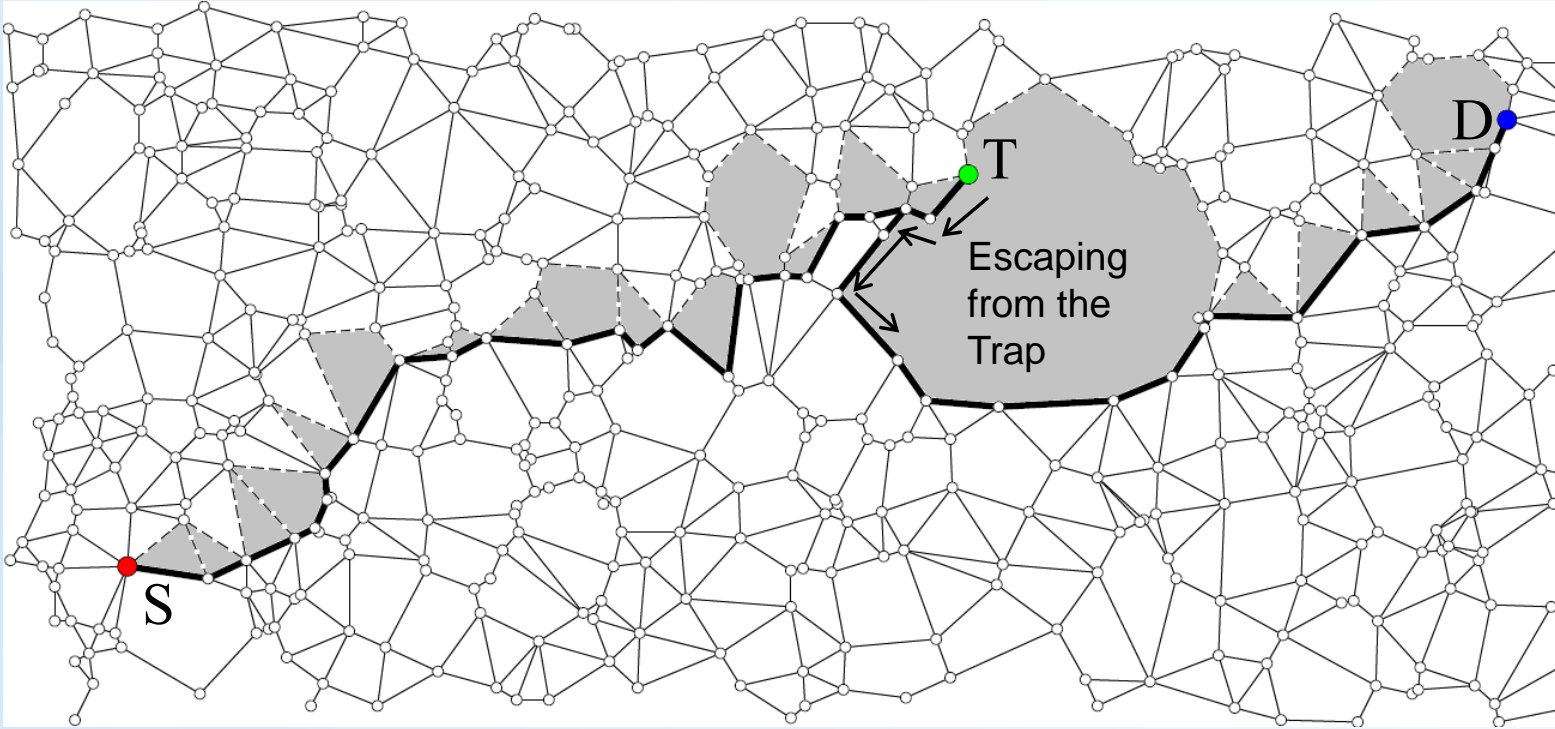

- Follow the Face of the Network to exit from the Trap (shown by directed line-segments)

\section{Identifying Redundant Nodes}

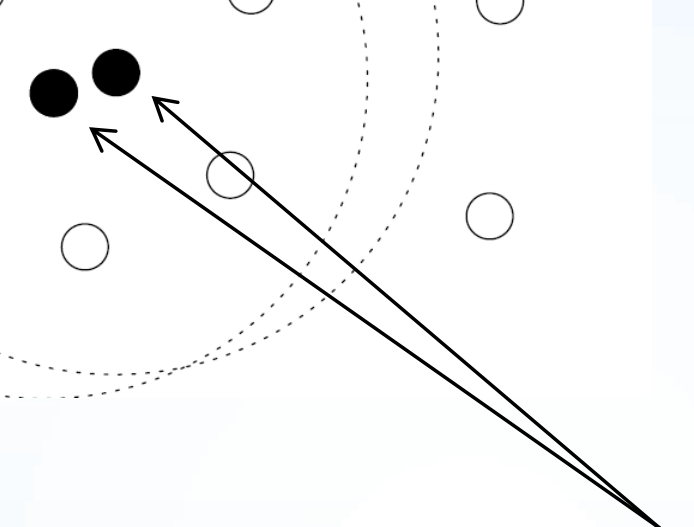

Two nodes close to each other are called equivalent if thei transmission range cover the same sub-set of nodes.

\section{Redundant Nodes in Clusters}

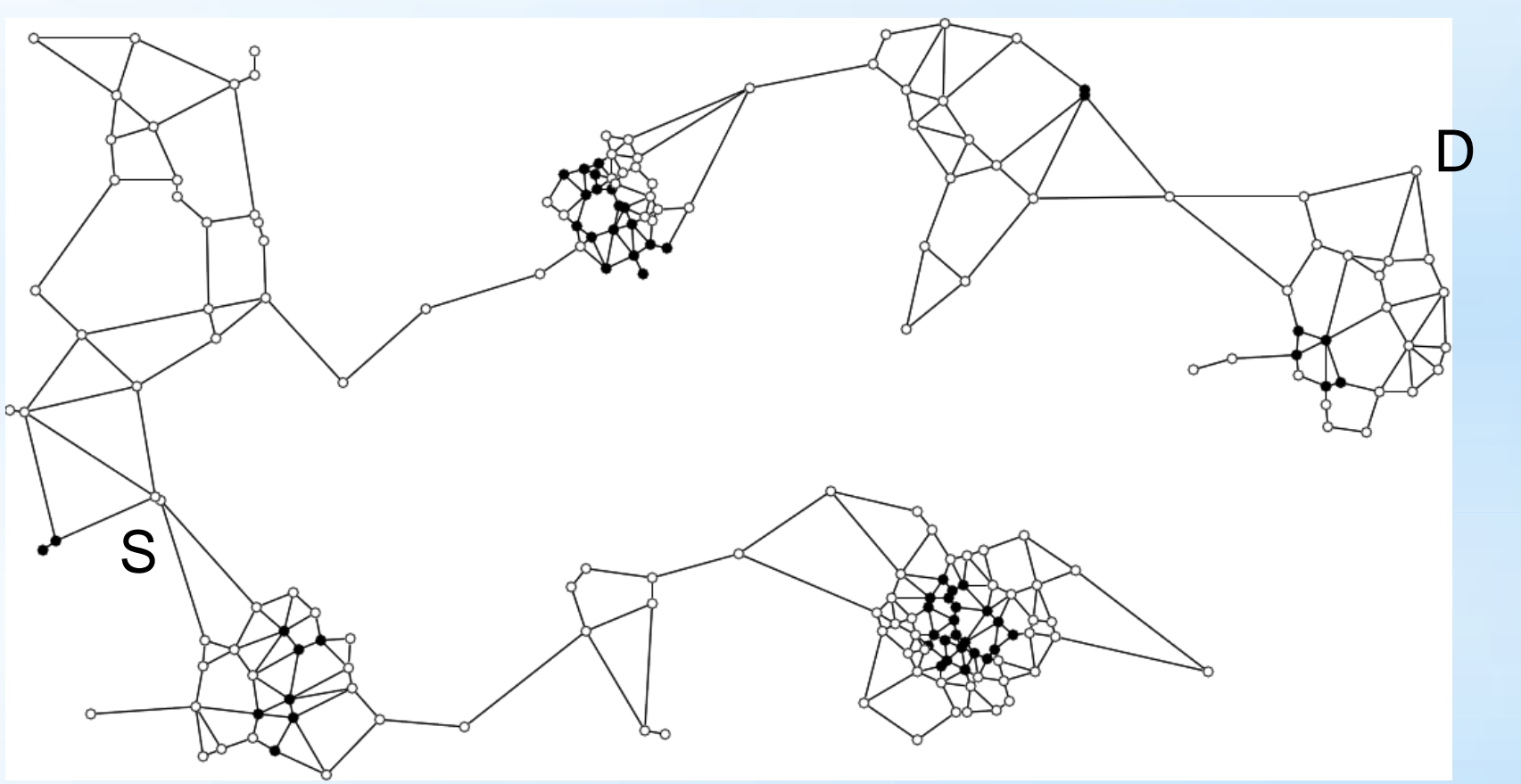

First Filtering Rule: Just keep representative node from redundant clusters (black-nodes)
Refined Network after Removing Redundant Members (un-connected!)

First Result (Our Finding): Connectivity between S and D is not compromised by removing redundant nodes.
Characterizing Floating Chains (enclosed by thick ellipse)
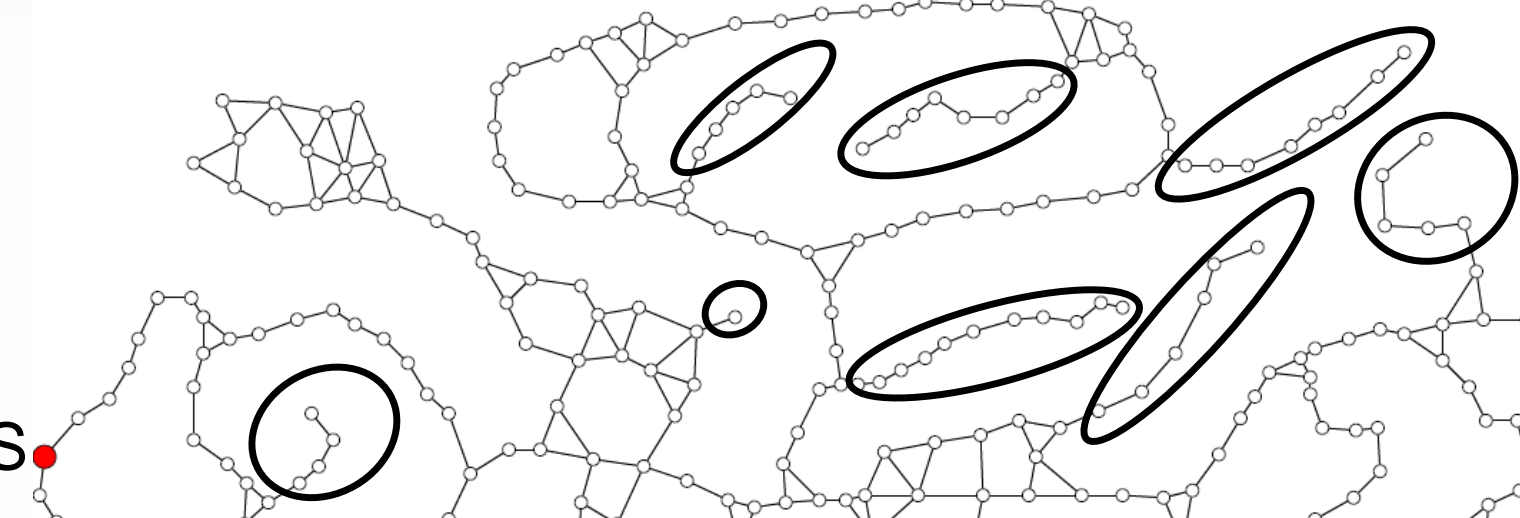

O

Second Result: Solo-Faced Floating Chains can be Filtered without compromising connectivity between $\mathrm{S}$ and $\mathrm{D}$
Network after Removing Floating-Chains

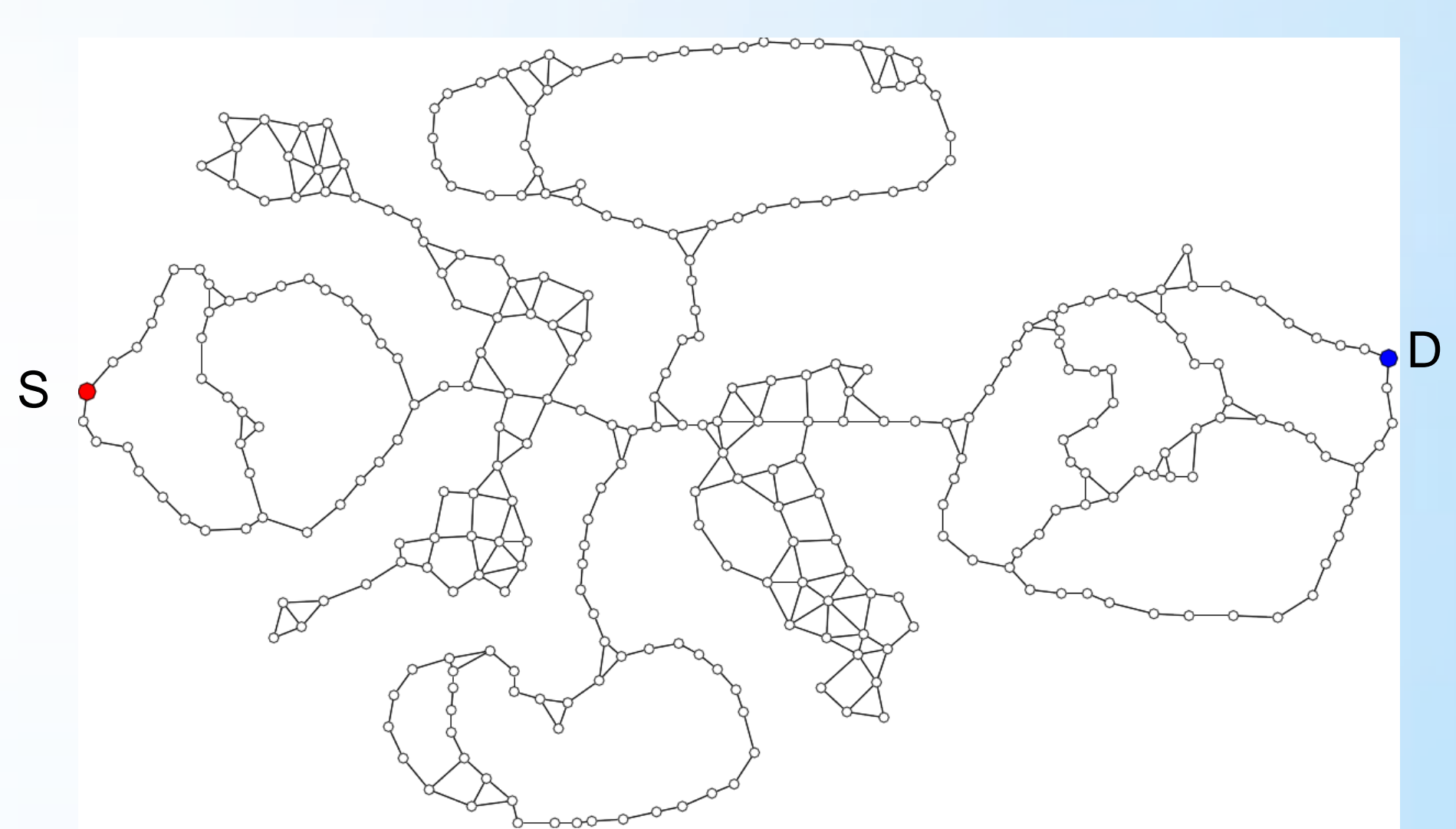

\section{Characterizing 2-connected components}

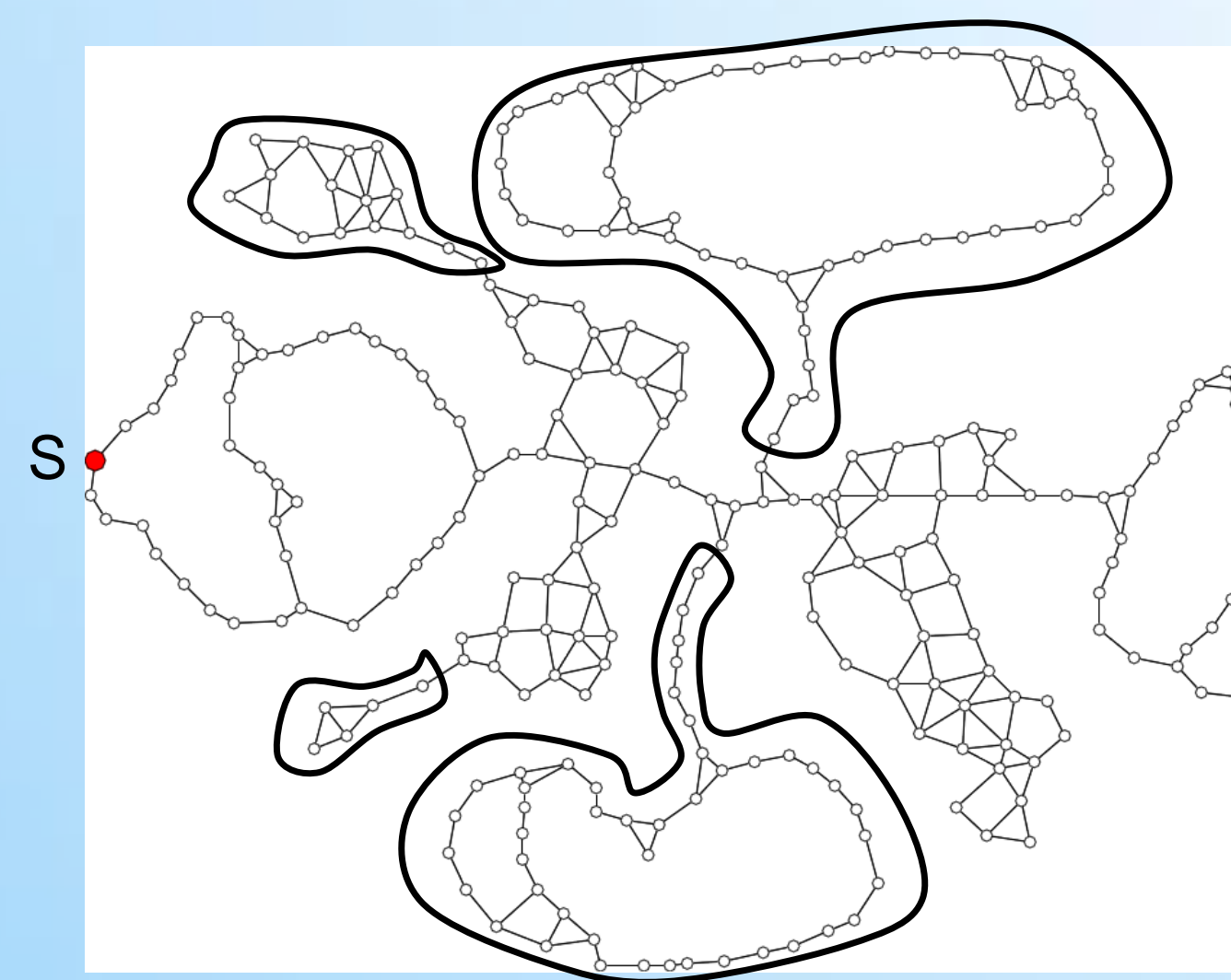

External 2-connected components can be removed withou compromising Network Connectivity between $\mathrm{S}$ and $\mathrm{D}$
Applying All Three Filtering Rules

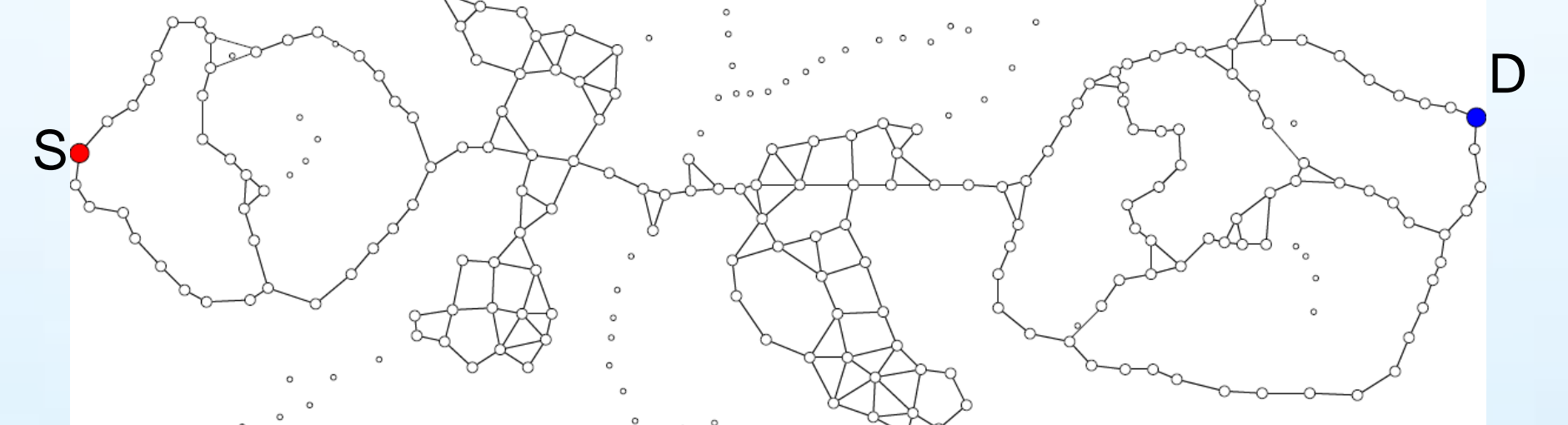

Network after filtering External 2-Connected Components. Filtered nodes are shown as tiny background dots.

\section{Java Implementation}

- Greedy Routing: Successful most of the time.

Hybrid Greedy-Face Routing: Guaranteed to be successful if there is a path from source to target

Node Filtering: Used for extending battery life.

\section{Future Work and Extensions}

- We are picking a redundant member from equivalent nodes arbitrarily

Develop Rules for picking appropriate node from equivalent cluster

Extensive simulated experimental investigation on randomly deployed sensor nodes

Apply Proposed Filtering Method on Real-World Sensor Network 